\title{
ESTRESSE EM FUNCIONÁRIOS DE UMA INSTITUIÇÃO DE ENSINO SUPERIOR: DIFERENÇAS ENTRE GÊNERO
}

\author{
Silvio Roberto Stefano \\ Universidade Estadual do Centro Oeste do Paraná \\ PPGA Universidade Estadual de Londrina \\ professor-silvio@ hotmail.com
}

Flavia Marcela Bonanato

Unicentro - PR

flavia.bona@gmail.com

Léo Raifur

Unicentro - PR

raifur@yahoo.com.br

Submissão: 15/09/2012

Aprovação: 27/01/2013

\section{Resumo}

São diversos impactos da relação entre estresse e trabalho, que afetam profundamente a qualidade de vida das pessoas tanto dentro quanto fora das organizações. O objetivo principal deste artigo foi identificar os fatores de estresse no ambiente de trabalho que podem ser causados por sua natureza e suas condições como: conflitos interpessoais entre o trabalho, as obrigações pessoais e às necessidades de auto realização no trabalho. A amostra compreendeu 78 funcionários, sendo 47 mulheres e 31 homens, foram aplicados questionários de autorelato aos pesquisados. O teste qui-quadrado $(p=0,757)$ em relação ao gênero demonstrou que a amostra é aderente. Foi utilizada a análise fatorial, onde os fatores foram extraídos com base na análise da variância total pelo método da Análise dos Componentes Principais (ACP) e foi utilizado o teste de Kaiser-Meyer-Olkin (KMO). Os resultados demonstraram que tanto homens quanto mulheres possuem taxas de estresse acima do recomendado, com variações específicas. As mulheres pesquisadas obtiveram maior incidência de problemas físicos relacionados ao estresse que os homens. A atividade física revelou-se uma importante forma da organização diminuir, ou até evitar o estresse entre os funcionários, implementando programas de prevenção dentro do próprio ambiente de trabalho.

Palavras-chave: estresse; qualidade de vida no trabalho; comportamento organizacional; gestão de pessoas. 


\begin{abstract}
There are several impacts of the relationship between stress and work that profoundly affect the quality of life of people both inside and outside of organizations. The main objective of this paper was to identify stressors in the work environment that may be caused by its nature and conditions as interpersonal conflicts between work, personal obligations and the needs of self-realization in work. The sample consisted of 78 employees, 47 women and 31 men, were administered self-report questionnaires to the respondents. The chi-square test $(p=0.757)$ in relation to gender showed that the sample is adherent. We used factor analysis, where the factors were extracted based on the analysis of the total variance by the method of Principal Component Analysis (PCA) was used and the test of Kaiser-Meyer-Olkin (KMO). The results showed that both men and women have rates above the recommended stress, with specific variations. The women surveyed had higher incidence of physical problems related to stress than men. Physical activity has proven to be an important form of organization reduce or even avoid stress among employees, implementing prevention programs within the workplace.
\end{abstract}

Key-words: stress, quality of work life, organizational behavior, human resources. 


\section{INTRODUÇÃO}

O estresse é uma doença que está cada vez mais presente nas organizações. Dessa forma, tem-se multiplicado os esforços de pesquisa de especialistas e de instituições no sentido de propor mecanismos que visem controlar os aspectos negativos no trabalho e sua prevenção permanente.

Segundo Pafaro e Martino (2004, p, 153) os problemas de saúde relacionados ao estresse podem se manifestar através de sintomas físicos, emocionais e comportamentais. Entre os sintomas físicos, os mais frequentes são as dores musculares, incluindo dor de cabeça, taquicardia, hipertensão arterial e problemas gastrintestinais. Sintomas emocionais: angústia, depressão, ansiedade, preocupação e insônia. Comportamentais: agressividade/passividade, distúrbios alimentares e mudanças na libido.

Essa relação entre estresse/trabalho se tornou tão comum que está tendendo para o trivial. Mas, não há dúvida de que o impacto do estresse na saúde e na qualidade de vida da pessoa pode ser tanto um estímulo e motivá-la a atingir seus objetivos como pode gerar situações devastadoras.

O interesse pelo estudo sobre as influências do gênero no campo do estresse ocupacional é recente, porém não menos importante. Observa-se que com relação ao gênero, poucas são as referências em estudos e bibliografias, e que a maioria dos estudos não menciona essa categoria.

De acordo com Lengnick-Hall (2003, p. 1043) "então, porque mais um livro sobre estresse? Acontece que, enquanto o estresse tem sido estudado por muitos anos e por diferentes perspectivas, uma área tem sido amplamente negligenciada: diferenças de gênero" (tradução dos autores).

Este tema, ainda de acordo com Lengnick-Hall (2003), pode revelar muito sobre estresse no trabalho e, assim, demonstra-se a importância de se responder aos seguintes questionamentos: Os agentes universitários estão com níveis de estresse? Existem diferenças entre os níveis de estresse, com relação ao gênero, em funções correlatas? Se estas diferenças existem, qual o comparativo entre os estressores e os fatores psicossociais com relação ao gênero?

O objetivo principal deste artigo foi identificar os fatores de estresse no ambiente de trabalho que podem ser causados por sua natureza e suas condições como: conflitos interpessoais entre o trabalho, as obrigações pessoais e às necessidades de auto realização no trabalho em uma Instituição de Ensino Superior. Usar-se-á, uma amostragem dos servidores, segundo gênero e função, utilizando questionário de auto relato sobre estresse no trabalho, resultando, então, num estudo sobre a influência do gênero em relação ao estresse ocupacional, comparando se existem diferenças entre os fatores psicossociais de risco e saúde mental e os estressores e fatores de apoio nas dimensões pessoa, do trabalho e social. 


\section{QUADRO TEÓRICO}

\subsection{CONCEITO E EVOLUÇÃO DO ESTRESSE}

De acordo com Silva e Martinez (2005), a palavra estresse tem origem latina e vem do verbo "stringo", que significa apertar, cerrar, comprimir, espreitar, restringir diminuir e reduzir. Ao consultar o dicionário Houaiss, tem-se a definição de estresse como o estado gerado pela percepção de estímulos que provocam excitação emocional e, ao perturbarem a homeostasia, levam o organismo a disparar um processo de adaptação caracterizado pelo aumento da secreção de adrenalina, com várias consequências sistêmicas.

O trabalho é essencial ao crescimento, sobrevivência e desenvolvimento do ser humano e, deveria, ainda, ser também fonte de prazer. O que se verifica é que uma parcela de culpa em relação à saúde do trabalhador pode ser dirigida ao trabalho, porque o modelo contemporâneo de trabalho ainda possui características da Revolução Industrial e do Taylorismo. De acordo com Côrrea e Saraiva (2000, apud MARTINS e OLIVEIRA, 2006, p. 230), é uma combinação de propostas do processo produtivo que traz agressões à saúde descendentes dos modelos tradicionais tayloristas e fordistas aliado a novos modelos.

$\mathrm{Na}$ tentativa de proporcionar maior conforto ao homem, o trabalho na contemporaneidade faz uso do avanço das tecnologias, tornando-o, assim, do ponto de vista físico, mais leve. No entanto, do ponto de vista psicológico, vem se configurando como algo pesado, árduo e, ainda, como fonte de competição. Segundo Martins e Oliveira (2006, p. 231), a busca pela sobrevivência vem fazendo de algumas empresas palco de disputas, em que o porte destas já não oferece garantia de permanência num mercado de trabalho fortemente competitivo.

De acordo com Azevedo e Kitamura (2006, p. 138) especificamente em relação ao trabalho, há um duplo reconhecimento por parte de estudiosos em relação ao estresse: por um lado, diversos aspectos do ambiente e da organização do trabalho podem ser geradores de estresse, e, sabe-se que um tipo de estresse está associado ao rebaixamento da qualidade de vida dos trabalhadores. Esse assunto será tratado mais profundamente no item causas do estresse.

\subsection{TIPOS DE ESTRESSE}

O estresse pode ser considerado como uma reação do organismo, confrontando situações que, de um modo ou de outro, o deixem desconfortável ou irritada, amedrontada, ou mesmo que deixe uma sensação de extrema felicidade.

Pode-se encontrar essa reação de estresse inserida na Classificação Internacional das Doenças (CID - 10, 1997). O CID - 10 inclui o estresse na categoria F43 - Reações ao estresse grave e transtornos de adaptação, e suas subcategorias F43.0 - Reação aguda ao estresse; F43.1 - Estado de estresse pós-traumático; F43.2 - Transtornos de adaptação; F43.8 - Outras reações ao estresse grave; F43.9 - Reação não especificada a um estresse grave.

Para melhor se entender o estresse como doença, reproduziu-se aqui a descrição do CID geral:

Esta categoria difere das outras na medida em que sua definição não repousa exclusivamente sobre a sintomatologia e a evolução, mas igualmente sobre a existência de um ou outro dos dois fatores causais seguintes: um acontecimento particularmente estressante desencadeia uma reação de "stress" aguda, ou uma alteração particularmente marcante na vida do sujeito, que comporta conseqüências desagradáveis e duradouras e levam a um transtorno de adaptação. Embora fatores de "stress" psicossociais ("life events") relativamente pouco graves possam precipitar a ocorrência de um grande número de transtornos classificados em outra

Revista Economia \& Gestão - v. 13, n. 31, jan./abr. 2013. 
parte neste capítulo ou influenciar-lhes o quadro clínico, nem sempre é possível atribuir-lhes um papel etiológico, quanto mais que é necessário levar em consideração fatores de vulnerabilidade, freqüentemente idiossincráticos, próprios de cada indivíduo; em outros termos, estes fatores não são nem necessários nem suficientes para explicar a ocorrência e a natureza do transtorno observado. Em contraste, para os transtornos reunidos aqui sob F43, admite-se que sua ocorrência é sempre a conseqüência direta de um "stress" agudo importante ou de um traumatismo persistente. O acontecimento estressante ou as circunstâncias penosas persistentes constituem o fator causal primário e essencial, na ausência do qual o transtorno não teria ocorrido. Os transtornos reunidos neste capítulo podem assim ser considerados como respostas inadaptadas a um "stress" grave ou persistente, na medida em que eles interferem com mecanismos adaptativos eficazes e entravam assim o funcionamento social.

Seley (1956, apud AZEVEDO e KITAMURA, 2006, p. 139-140) descreveu a Síndrome Geral de Adaptação como o conjunto de alterações não específicas que ocorrem no organismo quando ele é estressado. Consiste de três fases distintas:

Fase 1 - REAÇÃO DE ALARME: É equivalente a uma reação de emergência (...). As alterações fisiológicas desta fase se caracterizam por aumento da frequiência cardíaca, da pressão arterial e da frequiência respiratória, aumento da glicose no sangue, aumento da circulação de glóbulos vermelhos e brancos no sangue, dilatação das pupilas, bronco dilatação e ansiedade. Esta fase dura desde alguns dias até semanas.

Fase 2 - FASE DA RESISTÊNCIA: Ocorre quando a exposição do indivíduo aos fatores causadores de stress é duradoura (...). Caracteriza-se pelas seguintes alterações: aumento do córtex da glândula supra-renal, irritabilidade, insônia, oscilações do humor, diminuição da libido, gastrite e úlceras pépticas. Nesta fase as doenças de caráter psicossomático se instalam e tornam-se crônicas. Esta fase dura desde alguns meses até vários anos.

Fase 3 - FASE DA EXAUSTÃO: Nesta fase revelam-se as falhas dos mecanismos de adaptação. Há um retorno à reação de alarme, exaustão das possibilidades de resposta do organismo frente às demandas e à ocorrência de evento de alta gravidade que podem conduzir o organismo à morte.

Encontra-se, ainda, de acordo com Lipp (2000, apud SILVA; MARTINEZ, 2005, p. 54), a inclusão de uma nova fase, a quase-exaustão, fase essa intermediária entre a resistência e a exaustão, ficando dessa forma:

FASE DE QUASE-EXAUSTÃO: O Organismo está enfraquecido e não consegue se adaptar ou resistir ao estressor. As doenças começam a aparecer, tais como herpes simples, psoríase, picos de hipertensão e diabete, nos indivíduos geneticamente predispostos. Quando o estressor permanece atuante por muito tempo, ou quando muitas fontes de stress ocorrem simultaneamente, a reação do organismo progride para a fase da exaustão. 


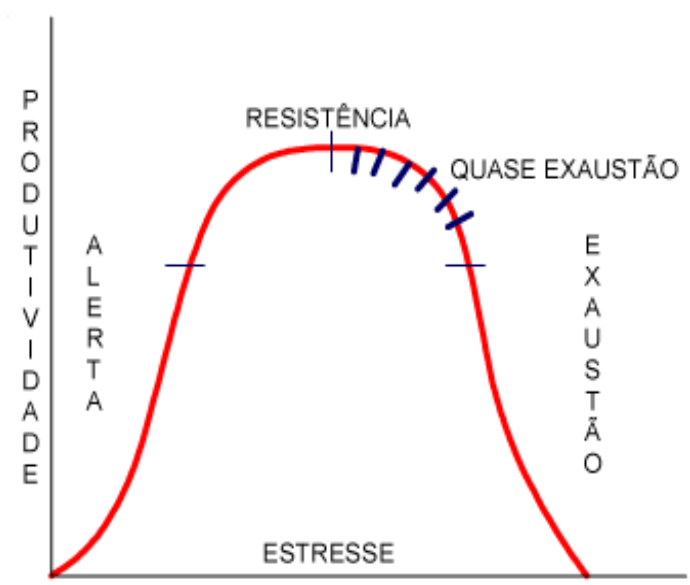

Figura 1: Produtividade do indivíduo em relação às fases do estresse Fonte: Pafaro e Martino (2004, p. 155). Adaptação total.

Não se pode esquecer que tanto situações consideradas ruins como as consideradas boas podem levar ao estresse. Dessa forma, o estresse pode ser ainda dividido, além das fases já mencionadas, em dois tipos: o eustress e o distress.

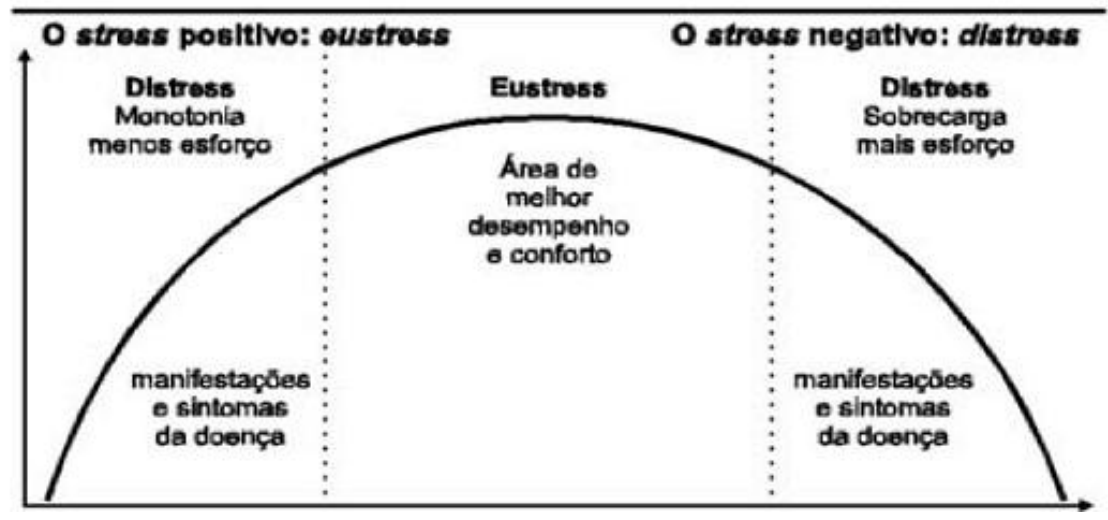

Figura 2: Tipos de estresse

Fonte: Lipp (1996) citado por Stefano; Roik (2005, p. 6). Adaptação total.

O eustress caracteriza-se pela tensão com equilíbrio entre esforço, tempo, realização e resultados, conhecido também como estresse positivo. $\mathrm{O}$ distress caracteriza-se pela tensão, com o rompimento do equilíbrio orgânico dinâmico por excesso ou falta de esforço, incompatível com o tempo, realização e resultados. É o estresse negativo.

\subsection{CAUSAS DE ESTRESSE}

Segundo Kyriacow e Sutcliffe (1981, apud LIPP; TANGANELLI, 2002, p. 539) o estresse ocupacional agrava-se quando há por parte do indivíduo a percepção das responsabilidades e poucas possibilidades de autonomia e controle. As dificuldades em adaptar-se a essas situações levam ao estresse.

Para Lipp e Tanganelli (2002) os estudos existentes terminam por se ater às necessidades de investimentos constantes em capacitações/treinamentos para os empregados, visando desenvolver as habilidades, assim como estimular o comprometimento dos mesmos com a organização, para que eles passem a se sentir valorizados e estimulados a enfrentar dificuldades maiores. Entretanto cabe ao gestor desenvolver políticas e programas de qualidade de vida no trabalho visando resgatar motivação, satisfação, saúde-segurança no trabalho. 
A preocupação das empresas com a qualidade de vida de seus colaboradores está fazendo com que as mesmas invistam cada vez mais em benefícios e melhores condições de trabalho. Diz ainda que "as organizações modernas começam a ter clareza das diferenças individuais entre homens e mulheres, que refletem no desempenho de cada um, além de compreender melhor a necessidade de uma análise completa da empresa, para garantir seu desenvolvimento" (DELBONI, 1997, p. 19).

No intuito de entender e solucionar os problemas relacionados ao estresse, e seus geradores, denominados estressores, ressalta-se a definição de Zillig (1998, p. 25-26) sobre o assunto: "Estressor é todo estímulo, real ou imaginário, que mobiliza o sistema de alarme de um organismo".

Ainda nessa linha, pode-se, ainda, verificar que são vários os estressores que afetam diariamente as pessoas, seja no campo pessoal como no profissional, trazendo várias consequências indesejáveis.

Para Santos (1995, p. 79) as mudanças que podem ocorrer no trabalho podem ser de todo tipo, como mudanças de instalações, de equipamento, de tecnologias, de volume de trabalho, de gerentes, de atitudes, política da companhia, táticas e assim seguindo. Cada mudança, independente de consulta prévia aos colaboradores, se manifesta como estressor dentro da empresa.

Segundo Lipp (1984, apud LIPP; TANGANELLI, 2002, p. 539):

$\mathrm{O}$ estresse pode ter origem de fontes externas e internas. As fontes internas estão relacionadas com a maneira de ser do indivíduo, tipo de personalidade e seu modo típico de reagir à vida. Os estressores externos podem estar relacionados com as exigências do dia-a-dia do indivíduo como os problemas de trabalho, familiares, sociais, morte ou doenças de um filho, perda de uma posição na empresa, não concessão de um objetivo de trabalho, perda de dinheiro ou dificuldades econômicas, notícias ameaçadoras, assaltos e violências das grandes cidades, etc.

McEwen e Lasley (2003) ainda afirmam que não se deve confundir estresse com sofrimento, e que os estressores estão aumentando, pois as pessoas estão diariamente sendo assediadas com mais informações do que um grupo de pessoas poderia acompanhar.

\subsection{ESTRESSE E GÊNERO}

Com relação à diferenciação do estresse em relação ao gênero e a preocupação das organizações, Delboni (1997, p. 19-20) esclarece que:

A conscientização da sobrecarga causada por uma jornada dupla de trabalho para a maioria das mulheres tem feito com que muitas empresas, independente da obrigatoriedade da lei, invistam na construção de modernas creches, administradas por pessoas especializadas, que garantem à funcionária tranquilidade quanto ao bem-estar de seus filhos e, conseqüentemente, permitem-lhe desempenhas melhor suas atividades. Da mesma forma, já podemos observar empresas construindo quadras de futebol em seus terrenos, para serem utilizadas por seus funcionários no final do expediente. Tal atitude garante a possibilidade de lazer, além de aumentar significativamente o espírito de equipe e o bom relacionamento entre as pessoas.

De acordo com Calais, Andrade e Lipp (2003) existem diferenças significativas entre os sexos quanto ao estresse, e estas devem ser analisadas sistematicamente, inclusive para prevenção dos efeitos negativos do mesmo.

Ainda nessa linha de pesquisa, afirma Rowlands (2008, p. 26) que "Novas pesquisas mostram que mulheres são mais suscetíveis ao estresse pelas relações no ambiente de trabalho enquanto que os homens são mais afetados pelas mudanças e pelo trabalho". (tradução dos autores)

Para Iwasaki; MacKay e Mactavish (2005 p. 3-4):

(...) as diferenças de gênero nas estratégias de enfrentamento poderiam surgir desde o início de socialização, que promove estereótipos das mulheres como emocionais, solidárias e dependentes, em comparação com os homens que são retratados como

Revista Economia \& Gestão - v. 13, n. 31, jan./abr. 2013. 
independentes, instrumentais, e racionais. As provas, porém, mostram que mulheres tendem a utilizar mais ativamente enfrentamentos comportamentais (...) do que os homens (Fielden \& Davidson, 2001). De igual forma, Gianakos (2000, 2002) constatou que as mulheres eram mais prováveis do que os homens de usar a ação direta de enfrentamento para lidar com o stress por trabalho mais longo e difícil. (tradução dos autores)

Outro grande diferencial entre homens e mulheres é o cuidado com os filhos, bem como o "trabalho de casa". Seguindo pesquisas de Posig e Kickil (2004, p. 374-375) nota-se essa diferenciação na cultura organizacional das empresas. 


\section{MÉTODO}

Segundo Cooper e Schindler (2011, p. 129), a pesquisa em questão caracteriza-se como sendo exploratório, com procedimentos de interrogação/comunicação por meio de questionário. Adicionalmente, trata-se de um estudo ex post facto, transversal e em condições de campo.

Com relação ao objetivo do estudo, é uma pesquisa causal, pois busca explicar as relações entre as variáveis entre si e entre grupos, como a relação entre fatores e sintomas físicos de estresse (COOPER; SCHINDLER; 2011, p. 129).

Quanto ao escopo, é um estudo estatístico, pois se apóia na análise quantitativa e visa deduzir as características de uma população a partir de uma amostra, com maior ênfase na amplitude do que na profundidade.

\subsection{Instrumento de coleta de dados}

A literatura internacional e nacional apresenta diversas escalas validadas para identificação e análise de estresse. Verificou-se www.periódicoscapes.gov.br em 20/12/2012, onde se obteve um total de 332 resultados válidos, em relação ao estresse de profissionais da saúde, de estudantes de diversos níveis, de pacientes de hospitais, de idosos e crianças, de trabalhadores de diversos setores específicos, etc. Na base se pesquisa SPELL® Scientific Periodicals Electronic Library http://www.spell.org.br/ em 19/12/2012, foram localizados 10 artigos relacionados ao estresse no trabalho. No PROQUEST em http://www.proquest.com.br/pt-BR/ em 21/12/2012 foram identificados 55.157 resultados de artigos sobre "stress" em seus diversos aspectos.

As escalas de avaliação de estresse laborais mais citadas foram: a escala de estresse no trabalho (EET) de Paschoal e Tamayo (2004) que foi desenvolvida e validada no Brasil e a escala de Lipp e Tanganelli (2002), além de escalas internacionais como: The Workplace Stress Scale ${ }^{\mathrm{TM}}$ Copyright (C) The Marlin Company, North Haven, CT, and the American Institute of Stress, Yonkers, NY e Job Stress Scale, entre outras.

Nesse estudo, foram utilizadas questões adaptadas das escalas de avaliação de estresse no trabalho de Lipp e Tanganelli (2002), Stefano e Roik (2005) e Wagner III e Hollenbeck (2006) para atender os objetivos da pesquisa.

$\mathrm{O}$ instrumento de pesquisa foi organizado em 2 blocos que procuraram verificar: a) fatores de risco, os estressores e fatores de apoio, dentro das dimensões social, pessoal e de trabalho, e; b) sintomas físicos de estresse.

O primeiro grupo foi composto de 28 questões em escala Likert com intensidade de 1 a 5 pontos (1. Concordo totalmente; 2. Concordo; 3. Não concordo nem discordo; 4. Discordo; 5. Discordo totalmente). O segundo grupo foi composto de 14 questões em escala de 3 pontos (1. Não tenho tido problemas; 2. Ocasionalmente; 3. Frequentemente).

\subsection{Caracterização da população e amostra}

O universo pesquisado é formado por 115 agentes universitários efetivos que desempenham funções operacionais ou ocupam funções/cargos de gerência ou supervisão. A amostra aleatória constitui-se de 78 questionários, sendo $60 \%$ do sexo feminino e $40 \%$ do sexo masculino. O teste qui-quadrado $(p=0,757)$ em relação ao gênero demonstrou que a amostra é aderente. Foram excluídos os agentes inativos, em férias ou em licença.

\subsection{Hipóteses}

O estudo testará as seguintes hipóteses.

$\mathrm{H}_{1}$ Os níveis de estresse em agentes universitários são baixos.

$\mathrm{H}_{2}$ Existem diferenças entre os níveis de estresse de homens e de mulheres. 
$\mathrm{H}_{3}$ Existe relação entre os fatores de estresse e os sintomas físicos de estresse.

\subsection{Tratamento dos dados}

Tendo em vista o número elevado de variáveis, foi utilizada a análise fatorial. Do primeiro grupo de questões foram excluídas 9 questões que estavam ligados mais a sintomas físicos que a fatores de estresse ou que já possuía uma outra variável medindo a mesma coisa. Dessa forma foram utilizadas 19 variáveis para o estudo.

Os fatores foram extraídos com base na análise da variância total pelo método da Análise dos Componentes Principais (ACP). Para retenção dos fatores obtidos na ACP foi utilizado o autovalor superior a 1. Foi utilizado o teste de Bartlett para validar a significância estatística das correlações entre as variáveis, com $p \leq, 000$. Para testar o grau de intercorrelações entre as variáveis e a adequação da amostra para a análise fatorial, será utilizado o teste de Kaiser-Meyer-Olkin (KMO) (tabela 1).

Tabela 1 - Interpretação dos resultados de KMO

\begin{tabular}{|c|c|}
\hline Kaiser-Meyer-Olkin & Interpretação \\
\hline $0,90 \longmapsto 1,00$ & Excelente \\
\hline $0,80 \longmapsto 0,90$ & Bom \\
\hline $0,70 \longmapsto 0,80$ & Mediano \\
\hline $0,60 \longmapsto 0,70$ & Medíocre \\
\hline $0,50 \longmapsto 0,60$ & Ruim (mas aceitável) \\
\hline$\longmapsto 0,50$ & Inaceitável \\
\hline
\end{tabular}

A carga fatorial absoluta para retenção da variável no fator é dada em função do tamanho da amostra, como demonstrado na tabela 2.

Tabela 2 - Cargas fatoriais significativas a partir do tamanho da amostra

\begin{tabular}{c|c}
\hline Carga fatorial & Tamanho da amostra \\
\hline 0,30 & 350 \\
\hline 0,35 & 250 \\
\hline 0,40 & 200 \\
\hline 0,45 & 150 \\
\hline 0,50 & 120 \\
\hline 0,55 & 100 \\
\hline 0,60 & 85 \\
\hline 0,65 & 70 \\
\hline 0,70 & 60 \\
\hline 0,75 & 50 \\
\hline
\end{tabular}

Fonte: Hair et. al. (2005, p. 107)

Para o caso do segundo grupo de questões, em função da escala limitar-se a 3 pontos, a análise fatorial será realizada sobre a matriz de correlação por pontos (Correlação de Spearman). Em função da má classificação na matriz (super ajuste) foram excluídas duas variáveis, de forma que foi procedida a análise com 12 variáveis.

\subsection{Teste das Hipóteses}

\subsubsection{Teste de $\mathrm{H}_{1}$}

Para identificar a existência de estresse na amostra estudada será utilizado o escore médio por fator, acrescido e decrescido do erro amostral do escore médio mais um desviopadrão.

Com base escala de intensidade desse grupo, têm-se os seguintes índices de estresse. Tabela 3 - Índice de estresse por fator 


\begin{tabular}{c|l}
\hline $\begin{array}{c}\text { Escore } \\
\text { Médio }\end{array}$ & $\begin{array}{c}\text { Interpretação do nível de } \\
\text { estresse }\end{array}$ \\
\hline 1,00 & Inexistente \\
\hline 1,50 & Muito baixo \\
\hline 2,00 & Baixo \\
\hline 2,50 & Pouco abaixo da média \\
\hline 3,00 & Médio \\
\hline 3,50 & Pouco acima da média \\
\hline 4,00 & Alto \\
\hline 4,50 & Muito Alto \\
\hline 5,00 & Altíssimo \\
\hline
\end{tabular}

Fonte: Elaborado pelos autores

\subsubsection{Teste de $\mathrm{H}_{2}$}

Para testar as diferenças entre os níveis de estresse do gênero masculino e feminino será utilizado o teste $t$-Student.

Para aceitação de $\mathrm{H}_{1}$ é necessário que $\mu$ (média dos escores do gênero masculino) seja significativamente $(p<0,05)$ diferente de $k$ ( média dos escores do gênero feminino). Para $\mu>$ $k$, têm-se que os homens tem nível de estresse mais elevado que as mulheres, e para $\mu<k$ tem-se a situação oposta.

\subsubsection{Teste de $\mathrm{H}_{3}$}

Os escores dos fatores de estresse do primeiro grupo serão correlacionados com os escores dos fatores dos sintomas físicos. A correlação será analisada com base no teste de Pearson. Para aceitação de $\mathrm{H}_{3}$ é necessário que $r$ seja significativo $(p<0,05)$. Espera-se que $r$ seja positivo, de forma a atestar que o aumento nos índices dos fatores estressores, aumentem os efeitos físicos consequentes da carga de estresse. 


\section{APRESENTAÇÃO E ANÁLISE DOS DADOS}

A amostra foi composta de 77 questionários distribuídos em dois grupos de perguntas. O primeiro grupo foi formado de 28 questões em escala de intensidade de cinco pontos, que procuraram medir os fatores de risco, os estressores e fatores de apoio dentro das dimensões social, pessoal e de trabalho. O segundo grupo continha 14 questões em escala de três pontos, que procuraram medir os sintomas físicos de estresse.

Dada a impossibilidade e a dificuldade de tratamento e interpretação das variáveis individualmente, procedeu-se a análise fatorial de onde se propôs a trabalhar com os fatores cujos autovalores foram superiores a 1.

Hair et. al. (2005, p. 98) recomendam pelo menos cinco vezes o número de observações em relação ao número de variáveis. No presente estudo está relação é de quatro vezes. Para evitar que os resultados obtidos estejam super ajustados (lixo dentro), a pertinência das variáveis também foi analisada do ponto de vista conceitual.

\begin{tabular}{|c|c|c|}
\hline \multicolumn{2}{|c|}{$\begin{array}{l}\text { Kaiser-Meyer-Olkin Measure of Sampling } \\
\text { Adequacy. }\end{array}$} &, 811 \\
\hline \multirow{3}{*}{$\begin{array}{l}\text { Bartlett's Test of } \\
\text { Sphericity }\end{array}$} & Approx. Chi-Square & 563,128 \\
\hline & $\mathrm{df}$ & 171 \\
\hline & Sig. & ,000 \\
\hline
\end{tabular}

Fonte: Elaborado pelos autores com o uso do SPSS.

No teste de Kaiser-Meyer-Olkin (tabela 4) de adequação da amostra foi obtido o valor de 0,811 , validando a adequação da análise fatorial a partir da análise das intercorrelações entre as variáveis (HAIR et. al., 2005, p. 98).

No teste de esfericidade de Bartlett (tabela 4) foi obtido $p=000$, validando que as correlações da variância total (matriz de correlação inteira) são significativas (HAIR et. al., 2005, p. 98).

Tabela 5 - Fatores e variância total explicada

\begin{tabular}{|c|c|c|c|c|c|c|c|c|c|}
\hline \multirow{2}{*}{$\begin{array}{c}\text { Compo- } \\
\text { nent }\end{array}$} & \multicolumn{3}{|c|}{ Initial Eigenvalues } & \multicolumn{3}{|c|}{$\begin{array}{l}\text { Extraction Sums of Squared } \\
\text { Loadings }\end{array}$} & \multicolumn{3}{|c|}{ Rotation Sums of Squared Loadings } \\
\hline & Total & $\begin{array}{c}\% \text { of } \\
\text { Variance }\end{array}$ & $\begin{array}{l}\text { Cumula- } \\
\text { tive } \%\end{array}$ & Total & $\begin{array}{c}\% \text { of } \\
\text { Variance }\end{array}$ & $\begin{array}{l}\text { Cumula- } \\
\text { tive } \%\end{array}$ & Total & $\begin{array}{c}\% \text { of } \\
\text { Variance }\end{array}$ & $\begin{array}{l}\text { Cumula- } \\
\text { tive } \%\end{array}$ \\
\hline 1 & 6,384 & 33,602 & 33,602 & 6,384 & 33,602 & 33,602 & 2,727 & 14,355 & 14,355 \\
\hline 2 & 1,720 & 9,053 & 42,655 & 1,720 & 9,053 & 42,655 & 2,619 & 13,784 & 28,138 \\
\hline 3 & 1,513 & 7,965 & 50,620 & 1,513 & 7,965 & 50,620 & 2,448 & 12,886 & 41,024 \\
\hline 4 & 1,403 & 7,382 & 58,002 & 1,403 & 7,382 & 58,002 & 2,293 & 12,070 & 53,095 \\
\hline 5 & 1,078 & 5,673 & 63,675 & 1,078 & 5,673 & 63,675 & 2,010 & 10,580 & 63,675 \\
\hline 6 & 891 & 4,687 & 68,362 & & & & & & \\
\hline 7 & ,773 & 4,069 & 72,431 & & & & & & \\
\hline 8 & ,756 & 3,978 & 76,409 & & & & & & \\
\hline 9 & 698 & 3,673 & 80,082 & & & & & & \\
\hline 10 &, 592 & 3,116 & 83,198 & & & & & & \\
\hline 11 &, 547 & 2,877 & 86,075 & & & & & & \\
\hline 12 &, 505 & 2,659 & 88,733 & & & & & & \\
\hline 13 & ,476 & 2,506 & 91,239 & & & & & & \\
\hline 14 &, 370 & 1,947 & 93,186 & & & & & & \\
\hline 15 &, 323 & 1,701 & 94,887 & & & & & & \\
\hline 16 & ,289 & 1,520 & 96,407 & & & & & & \\
\hline 17 & ,263 & 1,384 & 97,791 & & & & & & \\
\hline
\end{tabular}




\begin{tabular}{l|r|r|r|l|l|l|l|l|l}
\hline 18 &, 235 & 1,235 & 99,026 & & & & & & \\
\hline 19 &, 185 &, 974 & 100,000 & & & & & & \\
\hline
\end{tabular}

Fonte: Elaborado pelos autores com utilização do SPSS

A tabela 6 apresenta a matriz de componentes rotacionados, obtida pelo método de Análise de Componentes Principais e rotacionada pelo método Varimax com normalização de Kaiser. As comunalidades iniciais obtidas foram iguais a 1, e o poder de explicação da variância pelos fatores comuns é superior a $50 \%$ em todas as variáveis.

Tabela 6 - Matriz ${ }^{\mathrm{a}}$ de componentes rotacionados

\begin{tabular}{l|c|c|c|c|c|c}
\hline \multirow{2}{*}{\multicolumn{1}{c|}{ Variáveis }} & \multicolumn{5}{|c}{ Fatores } & Comuna- \\
\cline { 2 - 6 } & 1 & 2 & 3 & 4 & 5 & lidades \\
\hline Dist_Tar & $\mathbf{0 , 5 8 4}$ & 0,137 & 0,289 & 0,372 & 0,101 & 0,591 \\
\hline Contr_Trab & $\mathbf{0 , 5 0 0}$ & 0,437 & 0,311 & 0,177 & 0,121 & 0,584 \\
\hline F_Conf_Sup & 0,070 & 0,067 & $\mathbf{0 , 8 2 8}$ & 0,012 & 0,087 & 0,702 \\
\hline F_Info_Tar & 0,290 & 0,398 & 0,163 & 0,285 & 0,448 & 0,551 \\
\hline F_Com_Col & $\mathbf{0 , 6 1 6}$ & 0,195 & 0,361 & 0,212 & 0,147 & 0,615 \\
\hline Trat_Sup & 0,206 & 0,126 & $\mathbf{0 , 7 5 8}$ & 0,092 & 0,013 & 0,641 \\
\hline Inc_Tar & 0,496 & $-0,265$ & $-0,014$ & 0,406 & 0,396 & 0,639 \\
\hline Trab_Hs & $\mathbf{0 , 7 2 9}$ & 0,227 & $-0,120$ & $-0,060$ & 0,037 & 0,603 \\
\hline F_Com_Sup & 0,058 & 0,267 & $\mathbf{0 , 5 8 9}$ & 0,120 & 0,366 & 0,569 \\
\hline Discrim & 0,152 & $\mathbf{0 , 7 2 6}$ & 0,125 & $-0,008$ & 0,221 & 0,614 \\
\hline Isol_Org & 0,196 & $\mathbf{0 , 7 6 1}$ & $-0,040$ & 0,211 & $-0,133$ & 0,681 \\
\hline Desv_Sup & 0,191 & 0,196 & 0,406 & $\mathbf{0 , 6 0 7}$ & 0,208 & 0,651 \\
\hline Cresc_Car & $-0,187$ & 0,259 & 0,018 & $\mathbf{0 , 7 9 6}$ & 0,095 & 0,745 \\
\hline Tar_Inf & 0,227 & 0,100 & 0,050 & $\mathbf{0 , 8 0 6}$ & 0,075 & 0,720 \\
\hline Compet & 0,337 & $\mathbf{0 , 5 9 8}$ & 0,247 & 0,168 & 0,195 & 0,598 \\
\hline Ins_Tempo & $\mathbf{0 , 6 7 8}$ & 0,112 & 0,173 & $-0,065$ & 0,248 & 0,567 \\
\hline Sup_Evit_Resp & $-0,047$ & $\mathbf{0 , 6 1 8}$ & 0,407 & 0,219 & 0,029 & 0,599 \\
\hline Trab_dia_Pes & 0,279 & $-0,023$ & 0,218 & 0,157 & $\mathbf{0 , 6 5 7}$ & 0,582 \\
\hline Culp_Prob & 0,082 & 0,159 & 0,041 & 0,061 & $\mathbf{0 , 8 9 9}$ & 0,845 \\
\hline Eigenvalues & 2,727 & 2,619 & 2,448 & 2,293 & 2,010 & \\
\hline Var.Explicada & 14,35 & 13,78 & 12,89 & 12,07 & 10,58 & \\
\hline a) & & & & & \\
\hline
\end{tabular}

(a) Rotação convergida em 7 iterações (extraído com software SPSS)

A partir da matriz rotacionada foram selecionadas as variáveis com peso fatorial superior a 0,5. Hair et. al. (2005, p. 107) observam que o tamanho da amostra do presente estudo requereria a utilização do peso fatorial mínimo de 0,6 para que a significância $(p<$ $0,05)$ seja garantida. Foram incluídas quatro variáveis com pesos abaixo de 0,6 , sendo 0,598 , $0,589,0,584$ e 0,500 . Três dessas variáveis possuem valor muito próximo a 0,6 , e a inclusão delas deveu-se a avaliação conceitual e o fato de intrinsecamente refletirem o fator no qual foram classificadas. Foram excluídas as variáveis onde todos os pesos fatoriais absolutos apresentaram valor inferior 0,5 ou quando uma mesma variável pode ser explicada por mais de um fator. A denominação de cada um dos fatores apoiou-se na descrição conjunta das variáveis agrupadas.

Os cinco fatores selecionados, conforme demonstrado no quadro 1, explicam em torno de $64 \%$ da variância total da amostra. 
Quadro 1 - Descrição dos fatores em função do agrupamento das variáveis

\begin{tabular}{|c|c|c|}
\hline & Fator & Variáveis agrupadas \\
\hline 1 & $\begin{array}{l}\text { Ambiente e } \\
\text { organização do } \\
\text { trabalho }\end{array}$ & $\begin{array}{l}\text { - A forma como as tarefas são distribuídas em minha área tem me deixado nervoso; } \\
\text { - O tipo de controle existente no meu trabalho me irrita; } \\
\text { - A falta de comunicação entre mim e meus colegas de trabalho deixa-me irritado; } \\
\text { - Fico de mau humor por ter que trabalhar durante muitas horas seguidas; } \\
\text { - O tempo insuficiente para realizar meu volume de trabalho deixa-me nervoso; }\end{array}$ \\
\hline 2 & Competição & $\begin{array}{l}\text { - Fico irritado com a discriminação/favoritismo no meu ambiente de trabalho; } \\
\text { - Fico de mau humor por sentir-me isolado na organização; } \\
\text { - A competição no meu ambiente de trabalho tem me deixado nervoso; } \\
\text { - Fico incomodado por meu superior evitar me incumbir de responsabilidades } \\
\text { importantes. }\end{array}$ \\
\hline 3 & $\begin{array}{c}\text { Relação } \\
\text { Hierárquica }\end{array}$ & $\begin{array}{l}\text { - Tenho me sentido incomodado com a falta de confiança de meu superior sobre o } \\
\text { meu trabalho; } \\
\text { - Sinto-me incomodado por meu superior tratar-me na frente de colegas de trabalho; } \\
\text { - Sinto-me incomodado com a comunicação existente entre eu e meu superior; }\end{array}$ \\
\hline 4 & $\begin{array}{l}\text { Carreira e } \\
\text { realização } \\
\text { profissional }\end{array}$ & $\begin{array}{l}\text { - Fico irritado por ser pouco valorizado por meus superiores; } \\
\text { - As poucas perspectivas de crescimento na carreira têm me deixado angustiado; } \\
\text { - Tenho me sentido incomodado por trabalhar em tarefas abaixo do meu nível de } \\
\text { habilidade; }\end{array}$ \\
\hline & $\begin{array}{l}\text { Relacionamento } \\
\text { interpessoal }\end{array}$ & $\begin{array}{l}\text { - Trabalhar diariamente com pessoas me deixa irritado; } \\
\text { - Sinto que meus colegas me culpam por alguns problemas dentro do trabalho. }\end{array}$ \\
\hline
\end{tabular}

Fonte: Elaborado pelos autores

No quadro 1 os fatores foram categorizados em função das características das variáveis comuns a eles.

Quanto ao conjunto de questões que envolviam os sintomas físicos de estresse, o teste de KMO obtido foi de 0,804 e o teste de esfericidade de Bartlett foi significativo $(p=.000)$, validando a adequação da análise fatorial. A matriz foi obtida a partir Análise dos Componentes Principais e rotacionada pelo método varimax, convergida em 5 iterações. Foram retidos 3 fatores considerando-se os pesos fatoriais apresentados na tabela 7 .

Tabela 7 - Matriz de componentes rotacionados - sintomas físicos

\begin{tabular}{|c|c|c|c|c|}
\hline \multirow{2}{*}{ Componentes } & \multicolumn{3}{|c|}{ Fatores } & \multirow{2}{*}{$\begin{array}{r}\text { Comuna } \\
\text { lidades }\end{array}$} \\
\hline & 1 & 2 & 3 & \\
\hline Dor de Cabeça / Enxaqueca & $\mathbf{0 , 8 1 7}$ & $-0,051$ & $-0,005$ & 0,670 \\
\hline Insônia / fadiga & 0,673 & 0,341 & 0,031 & 0,570 \\
\hline Dor na parte inferior das costas & 0,591 & 0,349 & $-0,274$ & 0,546 \\
\hline Nervosismo & 0,497 & 0,641 & 0,100 & 0,667 \\
\hline Pesadelos & 0,274 & 0,712 & $-0,054$ & 0,585 \\
\hline Pressão arterial alterada & 0,634 & 0,171 & 0,281 & 0,510 \\
\hline Ingestão de álcool e automedicação & $-0,092$ & $-0,175$ & 0,725 & 0,564 \\
\hline Taquicardia & 0,627 & 0,250 & 0,438 & 0,648 \\
\hline Indigestão & 0,118 & 0,404 & 0,546 & 0,476 \\
\hline Crises respiratórias / Falta de ar & 0,269 & 0,338 & 0,576 & 0,518 \\
\hline Períodos de depressão & 0,084 & 0,776 & 0,130 & 0,626 \\
\hline Sentimento de raiva & 0,088 & 0,684 & 0,116 & 0,489 \\
\hline Eigenvalues & 4,326 & 1,347 & 1,196 & \\
\hline Variância explicada \% & 22,47 & 21,89 & 12,88 & \\
\hline
\end{tabular}

Fonte: Elaborado pelos autores (extraído com o software SPSS)

Os fatores identificados na tabela 7 ficaram assim discriminados: 
1) Distúrbios cardíacos e do sistema nervoso;

2) Distúrbios emocionais;

3) Distúrbios digestivos, respiratórios, automedicação e ingestão de álcool.

\subsection{Teste de $\mathrm{H}_{1}$}

Para analise dos níveis de estresse por fator foi calculado o escore médio por fator e, em seguida, foi encontrado o limite inferior (escore médio - erro amostral da média - 1 desvio - padrão) e o limite superior (escore médio - erro amostral da média - 1 desvio-padrão).

Tabela 8 - Estatística Descritiva - Fatores

\begin{tabular}{l|c|c|c|c|c|c|c|c}
\hline \multicolumn{1}{c|}{ Fator } & $\mathbf{N}$ & Mín. & Máx. & $\boldsymbol{\mu}$ & $\boldsymbol{\sigma}$ & $\boldsymbol{d}$ & $\boldsymbol{\mu}-\boldsymbol{d}-\mathbf{1 \sigma}$ & $\boldsymbol{\mu}+\boldsymbol{d}+\mathbf{1 \sigma}$ \\
\hline $\mathbf{1}$ Ambiente e organização do trabalho & 77 & 1,00 & 4,20 & 2,26 & 0,841 & 0,11 & 1,31 & 3,21 \\
\hline $\mathbf{2}$ Competição & 77 & 1,00 & 4,00 & 1,96 & 0,787 & 0,10 & 1,07 & 2,84 \\
\hline 3 Relação hierárquica & 77 & 1,00 & 4,67 & 1,69 & 0,757 & 0,10 & 0,84 & 2,55 \\
\hline 4 Carreira e realização profissional & 77 & 1,00 & 5,00 & 2,31 & 0,942 & 0,15 & 1,21 & 3,40 \\
\hline 5 Relacionamento interpessoal & 77 & 1,00 & 3,50 & 1,81 & 0,712 & 0,10 & 1,00 & 2,62 \\
\hline
\end{tabular}

Fonte: Elaborado pelos autores

A figura 3 ilustra os níveis de estresse em função dos fatores nos agentes universitários. Observa-se que em termos de escores médios dos fatores " 2. Competição", "3. Relação Hierárquica" e "5. Relacionamento interpessoal" apresentar escores classificados entre os níveis baixo e muito baixo. Os fatores " 1 . Ambiente e organização do trabalho" e "4. Carreira e realização profissional" ficaram classificados entre os níveis baixo e um pouco abaixo da média, sendo que seus limites superiores (acrescidos do erro amostral mais o desvio padrão) ultrapassam o nível médio.

Em sentido geral, os níveis de estresse identificados nos agentes universitários são baixos, destacando-se os "aspectos de ambiente e organização do trabalho" e "carreira e realização profissional” como os mais estressores.

1 Ambiente e organização do trabalho

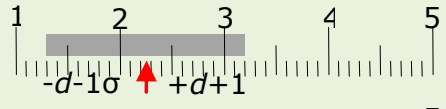

2 Competição

3 Relação hierárquica

4 Carreira e realização profissional

5 Relacionamento interpessoal

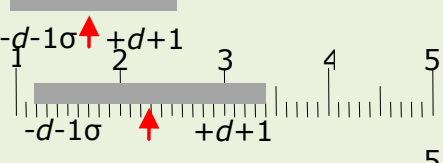

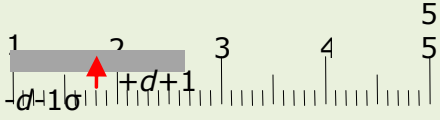

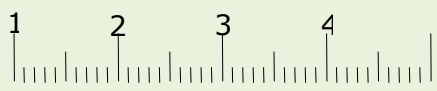

Figura 3 - Termômetro de fatores estressores

Fonte: Elaborado pelos autores

\subsection{Teste de $\mathrm{H}_{2}$}

Na comparação de cada um dos fatores em função do gênero, mediante a aplicação do teste $t$-Student, não foi encontrado diferença significativa, concluindo-se que na amostra 
pesquisada os fatores de estresse se manifestam de maneira equivalente tanto em homens como em mulheres.

\subsection{Teste de $\mathrm{H}_{3}$}

$\mathrm{A}_{3}$ assume que existe relação entre os fatores de estresse e os sintomas físicos de estresse. Para testá-la inicialmente foi construída a matriz de correlação de Pearson, conforme demonstrado na tabela 9.

Tabela 9 - Matriz de correlação de Pearson

\begin{tabular}{l|c|c|c|c|c|c}
\cline { 3 - 7 } \multicolumn{1}{c|}{ Sintomas físicos de estresse } & & $\begin{array}{c}\text { Ambiente e } \\
\text { organização } \\
\text { do trabalho }\end{array}$ & Competição & $\begin{array}{c}\text { Relação } \\
\text { Hierárquica }\end{array}$ & $\begin{array}{c}\text { Carreira e } \\
\text { realização } \\
\text { profissional }\end{array}$ & $\begin{array}{c}\text { Relação } \\
\text { interpessoal }\end{array}$ \\
\hline $\begin{array}{l}\text { Distúrbios cardíacos e do sistema } \\
\text { nervoso; }\end{array}$ & $r$ & $\mathbf{0 , 4 5 6}$ & 0,077 & 0,029 & 0,150 & $\mathbf{0 , 3 0 7}$ \\
\hline Distúrbios emocionais & $p$ & $\mathbf{0 , 0 0 0}$ & 0,503 & 0,805 & 0,193 & $\mathbf{0 , 0 0 7}$ \\
\hline $\begin{array}{l}\text { Distúrbios digestivos, } \\
\text { respiratórios, automedicação e } \\
\text { ingestão de álcool. }\end{array}$ & $r$ & $\mathbf{0 , 6 0 0}$ & $\mathbf{0 , 2 2 1}$ & $\mathbf{0 , 2 7 8}$ & $\mathbf{0 , 3 3 9}$ & $\mathbf{0 , 3 4 5}$ \\
\hline
\end{tabular}

$r$ : Correlação de Pearson; $p$ : significância ( $p$-value)

Fonte: Elaborado pelos autores

Observa-se que o fator "ambiente e organização de trabalho" está altamente correlacionado $(p<, 000)$ com os sintomas físicos de estresse. Não obstante, apesar de baixo, o respectivo fator de estresse apresentou escore médio ligeiramente acima dos demais.

Também é possível notar que os distúrbios emocionais estão relacionados, em maior ou menor grau, com todos os fatores de estresse.

O fator "carreira e realização profissional", que apresentou o escore médio mais elevado, evidenciou relação significativa apenas com distúrbio emocional.

Tabela 10 - Matriz de aceitação e rejeição de $\mathrm{H}_{3}$

\begin{tabular}{l|c|c|c|c|c}
\cline { 2 - 6 } \multicolumn{1}{c|}{ Sintomas físicos de estresse } & $\begin{array}{c}\text { Ambiente } \\
\text { organização } \\
\text { do trabalho }\end{array}$ & Competição & $\begin{array}{c}\text { Relação } \\
\text { Hieráquica }\end{array}$ & $\begin{array}{c}\text { Carreira e } \\
\text { realização } \\
\text { profissional }\end{array}$ & $\begin{array}{c}\text { Relação } \\
\text { interpessoal }\end{array}$ \\
\hline $\begin{array}{l}\text { Distúrbios cardíacos e do sistema } \\
\text { nervoso; }\end{array}$ & Aceita & Rejeita & Rejeita & Rejeita & Aceita \\
\hline Distúrbios emocionais & Aceita & Aceita & Aceita & Aceita & Aceita \\
\hline $\begin{array}{l}\text { Distúrbios digestivos, } \\
\text { respiratórios, automedicação e } \\
\text { ingestão de álcool. }\end{array}$ & Aceita & Rejeita & Rejeita & Rejeita & Rejeita \\
\hline
\end{tabular}

Fonte: Elaborado pelos autores 


\section{CONSIDERAÇÕES FINAIS}

A presente pesquisa investigou a incidência de estresse e os fatores deste no ambiente de trabalho que podem ser devidos à natureza do mesmo e suas condições, a conflitos interpessoais entre o trabalho e as obrigações, e necessidades pessoais de auto realização.

Neste estudo, o gênero masculino apresentou mais fatores psicossociais de estresse no trabalho do que o feminino. Mas, também o gênero masculino apresenta melhores resultados em fatores físicos que as mulheres. Verifica-se, então, que os respondentes do gênero feminino apresentam mais estressores externos do que os masculinos, estando, assim, mais propensos aos efeitos danosos do estresse.

Segundo Areias e Guimarães (2004, p. 261):

Grande número de mulheres vive numa cultura competitiva, ambiente que pode favorecer o aparecimento de sentimentos de inadequação e baixa auto-estima. Além disso, conflitos não resolvidos, auto-repressão e expectativas podem produzir padrões de estresse e a ocorrência de doenças psicossomáticas com uma frequência maior.

Isso explica, de certa forma, os dados obtidos nas análises, em que os problemas físicos estão mais presentes nos respondentes do gênero feminino. Assim, estudos sobre estresse nas organizações tornam-se cada vez mais relevantes, uma vez que gestores podem utilizá-los para a definição de políticas e estratégias de redução do estresse organizacional e suas consequências.

Neste sentido, analisando do ponto de vista gerencial, percebe-se a importância de se construir na organização um clima de suporte social, num ambiente relacional positivo no trabalho, tanto entre colegas como também entre subordinados e gerentes. De acordo com Silva; Lima e Tamayo (2002, p. 10-11):

Na prevenção do estresse consideram-se três tipos ou níveis de intervenção. A prevenção primária consiste em realizar ações destinadas a modificar ou eliminar fontes de estresse no trabalho, a secundária pode ser ilustrada pela gestão do estresse e a terçaria por programas organizacionais de assistência médico-psicológica para os empregados. A gestão do clima é um exemplo de prevenção primária.

A Qualidade de Vida no Trabalho - QVT refere-se aos aspectos favoráveis e desfavoráveis no ambiente de trabalho para as pessoas. Tentando solucionar esse problema, as organizações vivem um paradoxo. De um lado, as organizações precisam do alcance de resultados, ótimos produtos/serviços e baixo custo. De outro, os funcionários necessitam de ótimas condições de trabalho e um clima organizacional favorável.

Dessa forma, deve-se dar prioridade à valorização da condição do servidor, mas sempre tendo foco na organização, conseguindo, assim, conciliar essas duas demandas. São indicadas medidas para gerenciar o estresse na instituição analisada tais como: programas estruturados de qualidade de vida no trabalho, incluindo aspectos biológicos, psicológicos, sociais e organizacionais.

Entre as medidas apontam-se: o planejamento, a organização e administração do tempo; melhoria das relações interpessoais; canais abertos de comunicação; reuniões periódicas de feedback; programas anti-tabagismo; contra o alcoolismo; diabetes; obesidade; redução do ruído no ambiente de trabalho; atividades físicas no ambiente de trabalho como a ginástica laboral e fora como natação ou outras atividades com recomendação médica, entre outras.

Segundo Martinez e Latorre (2009, p. 770) os estudos sobre estresse no ambiente de trabalho são importantes, pois permitem:

identificar aspectos que devem ser considerados na concepção e desenvolvimento de medidas visando a preservação da capacidade para o trabalho, com ênfase no controle do estresse relacionado aos fatores do ambiente psicossocial do trabalho e na promoção, proteção e recuperação da saúde por meio da prevenção e tratamento

Revista Economia \& Gestão - v. 13, n. 31, jan./abr. 2013. 
da dependência do álcool, incentivo à prática de atividade física e controle de peso.

De toda forma, poucos estudos foram identificados na literatura onde o foco do estresse em relação ao gênero fosse analisado. Assim, novas pesquisas sobre o assunto são necessárias para que haja comparação de resultados e elementos, ajudando assim a fundamentar ou refutar hipóteses apresentadas.

Ressalte-se aqui, também, o caráter exploratório dessa pesquisa, como forma de incentivar outras análises nessa linha e consolidar a importância do estudo do estresse nas instituições, bem como da promoção de programas de qualidade de vida no trabalho, valorizando, também, as atividades físicas, sociais e psicológicas. 


\section{REFERÊNCIAS}

AREIAS, M. E. Q.; GUIMARAES, L. A. M. Gênero e estresse em trabalhadores de uma universidade pública do estado de São Paulo. Psicologia em estudo, Maringá, v. 9, n. 2, 2004. Disponível em: < http://www.scielo.br/pdf/pe/v9n2/v9n2a11 >. doi: 10.1590/S141373722004000 200011. Acesso em 31 Ago 2012.

AZEVEDO, V. A. Z. de; KITAMURA, S. Stress, trabalho e qualidade de vida. In: VILARTA, Roberto. Qualidade de vida e fadiga institucional / Roberto Vilarta, Teresa Helena Portela Freire de Carvalho, Aguinaldo Gonçalves e Gustavo Luis Gutierrez (Organizadores). Campinas, SP: IPES Editorial, 2006, p. 137-150.

CALAIS, S. L.; ANDRADE, L. M. B. de; LIPP, M. E. N. Diferenças de sexo e escolaridade na manifestação de Stress em adultos jovens. Psicologia: Reflexão e Crítica, Porto Alegre, v. 16, n. 2, 2003. Available from <http://www.scielo.br/scielo.php?script=sci_arttext\&pid=S0102$79722003000200005 \& \operatorname{lng}=$ en\&nrm=iso >. doi: 10.1590/S0102-79722003000200005. Acesso em 15 Abr. 2012.

CID-10. Classificação Estatística Internacional de Doenças e Problemas Relacionados à Saúde. Décima Revisão, Versão 2008, Volume I. Disponível em: < http://www.datasus.gov. br/cid10/v2008/>cid10.htm. Acesso em 01 Jun 2012.

COOPER, D. R.; SCHINDLER, P. S. Métodos de pesquisa em administração. 10. ed. Porto Alegre: Bookman, 2011.

DELBONI, T. H. Vencendo o stress. São Paulo: Makron Books, 1997.

HAIR, J. F. Jr et. al. Análise multivariada de dados. 5 ed. Porto Alegre: Bookman, 2005. HOUAISS. Dicionário Eletrônico da Língua Portuguesa. Produzido e Distribuído por Editora Objetiva Ltda. Versão 1.0.10 - Março de 2006.

IWASAKI, Y.; MACKAY, K.; MACTAVISH, J. 2005. Gender-Based Analyses of Coping with Stress among Professional Managers: Leisure Coping and Non-Leisure Coping. Journal of Leisure Research 37, no. 1 (January 1): 1-28. http://www.proquest.com. Acesso em 8 Nov 2011.

LIPP, M. E. N.; TANGANELLI, M. S. Stress e qualidade de vida em Magistrados da Justiça do Trabalho: diferenças entre homens e mulheres. Psicologia: Reflexão e Crítica, Porto Alegre, v. 15, n. 3, 2002, p. 537-548. Disponível em: < http://www.scielo.br/pdf/prc/v15n3/a08v15n3.pdf >. doi: 10.1590/S010279722002000300008. Acesso em 03 Set 2012.

LENGNICK-HALL, M. L. Gender, Work Stress, and Health. Review of medium_being_reviewed title_of_work_reviewed_in_italics. Personnel Psychology, 56(4), 1043-1045, 2003. Retrieved October 21, 2008, from ABI/INFORM Global database. (Document ID: 523236681). Acesso em 21 Out 2011.

MAROCO, J. Análise estatística - com utilização do SPSS. 3. ed. Lisboa (Portugal): Silabo, 2007.

MARTINEZ, M. C.; LATORRE, M. do R. D. de O. Fatores associados à capacidade para o trabalho de trabalhadores do Setor Elétrico. Cad. Saúde Pública [online]. 2009, vol.25, n.4 [cited 2009-06-05], pp. 761-772. Available from: <http://www.scielo.br/scielo.php?script=sci_arttext\&pid=S0102311X2009000400007\&lng=en\&nrm=iso>. ISSN 0102-311X. doi: 10.1590/S0102311X2009000400007. Acesso em 05 Jun 2012. 
MARTINS, A. C. A.; OLIVEIRA, G. de. Trabalho: Fonte de prazer e sofrimento e as práticas orientais. In: VILARTA, Roberto. Qualidade de vida e fadiga institucional / Roberto Vilarta, Teresa Helena Portela Freire de Carvalho, Aguinaldo Gonçalves e Gustavo Luis Gutierrez (Organizadores). Campinas, SP: IPES Editorial, 2006, p. 229-241. MATOS, F. R. N.; LIMA, A. C. . Uma investigação empírica sobre o stress no ambiente acadêmico. In: VII SEMEAD - Seminários em Administração da Universidade de São Paulo, 2004, São Paulo. Anais do VII SEMEAD, 2004. Disponível em: < http://www.ead.fea.usp.br/Semead/7semead/paginas/artigos\%20recebidos/RH/RH54__Uma_investiga\%E7\%E3o_emp\%EDrico_stress.PDF>. Acesso em 08 Nov 2011.

McEWEN, B. S; LASLEY, E. N. O fim do stress como nós o conhecemos. Rio de Janeiro: Nova Fronteira, 2003.

PAFARO, R. C.; DE MARTINO, M. M. F. Estudo do estresse do enfermeiro com dupla jornada de trabalho em um hospital de oncologia pediátrica de Campinas. Revista da Escola de Enfermagem da USP, São Paulo, v. 38, n. 2, June 2004 . Disponível em < http://www.scielo.br/scielo.php?script=sci_arttext\&pid=S0080-62342004000200005\&lng= en \&nrm=iso>. doi: 10.1590/S0080-62342004000200005. Acesso em 27 Fev 2012.

POSIG, M.; KICKUL, J. Work-role Expectations and Work Family Conflict: Gender Differences in Emotional Exhaustion. Women in Management Review. 2004 Oct 1;19(7/8): 373-386. In: ABI/INFORM Global [database on the Internet] [cited 2009 Jan 11]. Available from: http://www.proquest.com/; Document ID: 774546121. Acesso em 11 Jan 2012.

ROSA, A. ; RODRIGUES, A. L. A.; SANTOS, F. V. dos . A contribuição da atividade física na Qualidade de Vida no Trabalho. In: VII Seminários em Administração da FEA/USP, 2004, São Paulo. Anais do VII SEMEAD. São Paulo: FEA/USP, 2004. Disponível em < http://www.ead.fea.usp.br/Semead/7semead/paginas/artigos\%20recebidos/RH/RH15__Contribui\%E7\%E3o_atividade_fisica_no_trabal.PDF>. Acesso em 27 Jan 2012.

ROWLANDS, R. Stress agender. Occupational Health, Jun 2008, p. 26-27. Disponível em: < http://www.personneltoday.com/articles/2008/09/01/46045/mental-health-and-genderstress-agender.html>. Acesso em: 27 jan 2012.

SANTOS, O. de A. Ninguém morre de trabalhar: o mito do stress. $3^{\text {a }}$ ed., São Paulo: Textonovo, 1995.

SILVA, A. V. Da; LIMA, D.; TAMAYO, A. Impacto do clima organizacional sobre o estresse no trabalho. In: $26^{\circ}$ ENANPAD 2002. Salvador BA, 2002. Anais, p.59-46.

Disponível em: < http://www.anpad.org.br/enanpad/2002/dwn/enanpad2002-cor-1624.pdf>. Acesso em: 03 Set 2012.

SILVA, E. A. T. da, MARTINEZ, A. Diferença em nível de stress em duas amostras: capital e interior do estado de São Paulo. Estudos de Psicologia (Campinas). [online]. mar. 2005, vol.22, no.1 [citado 15 Abril 2009], p.53-61. Disponível em: < http://www.scielo.br/pdf/estpsi/v22n1/v22n1a06.pdf>. ISSN 0103-166X. Acesso em: 15 de abril de 2012.

STEFANO, S. R., ROIK, A. Estresse Bancário e Sistemas de Administração de Pessoas: um estudo exploratório na cidade de Irati-Pr. In: SEMINÁRIOS EM ADMINISTRAÇÃO SEMEAD, 8. 2005, São Paulo. Anais (São Paulo: s/n. 2005). p.1-15.

WAGNER III, J. A., HOLLENBECK, J. R. Comportamento organizacional. Tradução Cid Knipel Moreira; São Paulo: Saraiva, 2006.

ZILLIG, C. Dose o stress: tempere a vida. Blumenau: EKO, 1998. http://www.datasus.gov.br/cid10/v2008/descrcsv.htm. Acesso em 01 Jun 2012. 